\title{
Editorial
}

\section{Comorbidity and beyond: pre-transplant clinical assessment}

\author{
Bone Marrow Transplantation (2005) 36, 473-474. \\ doi:10.1038/sj.bmt.1705042
}

'And in the end it's not the years in your life that count. It's the life in your years.'

Abraham Lincoln

Suitability for transplant has historically employed clinical judgment and single organ comorbidity testing, such as pulmonary, cardiac and hepatic function. In the era of intensive myeloablative only conditioning, such evaluation may have proven adequate to restrict transplant to fairly young and fit patients. Advances in supportive care, reduced-intensity conditioning and more tolerable GVHD prophylaxis have resulted in reduced acute morbidity and mortality after allogeneic hematopoietic transplantation in older and/or more ill patients. ${ }^{1}$ Extending this potentially curative modality to older patients represents a major advance as hematologic malignancies occur more commonly and are more refractory with advancing age. The mean age in recent trials with reduced-intensity approaches is often over 50 years of age. With less restrictive eligibility, particularly noteworthy for allogeneic hematopoietic cell transplantation, new challenges have arisen in evaluating an older and more heterogeneous population.

Age is perhaps the most commonly used surrogate for health status. Although chronologic aging parallels biologic aging, age by itself varies tremendously among individual patients. While comorbidity and disability rise with advancing age, ${ }^{2}$ the age-specific prevalence has decreased. In geriatrics, age plays little role in summarizing overall health but rather the assessment entails a variety of domains including comorbidity as well as functional status, mental status, nutritional status, emotional conditions and geriatric syndromes. Such comprehensive geriatric evaluations have increasingly been applied to oncology patients. ${ }^{3}$ Transplant physicians are just beginning to navigate this unfamiliar territory.

Comorbidity scales that provide relative weights to nondisease-related medical conditions permitting a summary score have recently been investigated for allogeneic hematopoietic transplant recipients. In their timely review, Dr Alamo et $a l^{4}$ concisely summarize the literature on pretransplant comorbidity assessment. The literature on single organ comorbidity testing reveals surprisingly limited utility, recognizing significant organ compromise may have precluded transplant, particularly in the era of myeloablative only conditioning. The review serves as an excellent reference and foundation to direct future research.

The Seattle Group reported on comorbidity as scored by the Charlson Comorbidity Index (CCI) with related and unrelated donors after both ablative and nonablative regimens. ${ }^{5,6}$ Higher CCI scores were associated with more grade IV toxicity as well as nonrelapse mortality (in multivariate analysis) among recipients of related and unrelated allografts. Our group and the MD Anderson Cancer Center have reported similar findings in abstract form. ${ }^{7,8}$ In addition, we found that a more sensitive comorbidity scale detected more comorbidity while maintaining prognostic power. Many limitations remain in the aforementioned studies as the comorbidity scales employed do not typically include information on prior infections (eg prior fungal infection) or grading of the comorbidities (eg degree of heart failure). Nevertheless, even with simple, crude, retrospective measures, comorbidity scales have considerable ability to predict transplant morbidity and mortality.

The fledgling area of geriatric oncology has also paved the road to demonstrating the independent importance of functional measures in addition to comorbidity. ${ }^{9}$ Performance status is a very simple, widely employed measure of functional status. In the transplant setting, performance status has shown appreciable prognostic influence for transplant-related mortality and overall survival, ${ }^{10-12}$ although data are limited comparing comorbidity and functional measures. Our preliminary data demonstrate that performance status confers independent if not superior prognostic power to comorbidity assessment. ${ }^{7}$ Alamo et $a l^{4}$ astutely note in their review the critical importance of employing functional measures in addition to comorbidity determination in future studies. The major limitation in using such tools in the transplant setting will be identifying tools that further stratify patients with preserved performance status, such as those with Karnofsky performance status scores above $70 \%$.

The inevitable question that arises is what is the value of a detailed pre-transplant health status evaluation? Several obvious benefits may accrue. Simplified scores that better capture patients' health status offer promise to more accurately and precisely estimate transplant-related morbidity and mortality. A score should help determine not only the risk/benefit ratio of transplant, but possibly enable a risk-adapted approach where transplant regimens may be tailored to both disease and health status. For example, a score may help establish the regimen tolerability of a 55 -year-old patient with acute myelogenous leukemia in second complete remission, allowing selection of a regimen intensive enough to minimize disease relapse while having acceptable transplant-related complications. An additional benefit of better defining the patient population would be to facilitate comparisons and inferences from studies. The notion that aside from age, disease and transplant factors adequately describe a patient population undergoing allogeneic hematopoietic transplantation requires serious scrutiny. Finally, by raising awareness to functional limitations or comorbidities, medical care directed at the limitations identified may be implemented.

The available data suggest future studies should focus on a prospective and comprehensive evaluation of nondisease 
factors to predict transplant tolerance, incorporating at least comorbidity and functional measures, if not emotional, social and nutritional domains. Future studies hold promise to enable an individualized approach to determine the risk/benefit ratio of transplant for patients and physicians alike.

AS Artz

Hematology/Oncology, University of Chicago, USA

\section{References}

1 Van Besien K, Artz A, Stock W. Unrelated donor transplantation over the age of 55. Are we merely getting (b)older? Leukemia 2005; 19: 31-33.

2 Yancik R, Havlik RJ, Wesley MN et al. Cancer and comorbidity in older patients: a descriptive profile. Ann Epidemiol 1996; 6: 399-412.

3 Balducci L. Geriatric oncology. Crit Rev Oncol Hematol 2003; 46: $211-220$.

4 Alamo J, Shahjahan M, Lazarus HM et al. Comorbidity indices in hematopoietic stem cell transplantation: a new report card. Bone Marrow Transplant 2005; 36: 475-479.

5 Sorror ML, Maris MB, Storer B et al. Comparing morbidity and mortality of HLA-matched unrelated donor hematopoietic cell transplantation after nonmyeloablative and myeloablative conditioning: influence of pretransplantation comorbidities. Blood 2004; 104: 961-968.
6 Diaconescu R, Flowers CR, Storer B et al. Morbidity and mortality with nonmyeloablative compared with myeloablative conditioning before hematopoietic cell transplantation from HLA-matched related donors. Blood 2004; 104: 1550-1558.

7 Pollyea DA, Artz AS, Stock W et al. Clinical predictors of transplant related mortality after reduced intensity conditioning allogeneic stem cell transplantation (RIST). Blood 2004; 104 (abstract 1145).

8 Shahjahan M, Alamo J, de Lima M, Khouri I. Effect of comorbidities on allogeneic hematopoietic stem cell transplant outcomes in AML/MDS patients in first complete remission. Biol Blood Marrow Transplant 2004; 10 (Supplement) 1 .

9 Extermann M, Overcash J, Lyman GH et al. Comorbidity and functional status are independent in older cancer patients. J Clin Oncol 1998; 16: 1582-1587.

10 van Besien K, Sobocinski KA, Rowlings PA et al. Allogeneic bone marrow transplantation for low-grade lymphoma. Blood 1998; 92: 1832-1836.

11 Giralt S, Thall PF, Khouri T et al. Melphalan and purine analog-containing preparative regimens: reduced-intensity conditioning for patients with hematologic malignancies undergoing allogeneic progenitor cell transplantation. Blood 2001; 97: 631-637.

12 Sayer HG, Kroger M, Beyer $\mathbf{J}$ et al. Reduced intensity conditioning for allogeneic hematopoietic stem cell transplantation in patients with acute myeloid leukemia: disease status by marrow blasts is the strongest prognostic factor. Bone Marrow Transplant 2003; 31: 1089-1095. 